\title{
Article \\ Discrepant End-Tidal Concentrations of Sevoflurane at the Same A-Line Autoregressive Index Level during Induction of General Anesthesia: An Observational Study
}

\author{
Che-Hao Hsu ${ }^{1,2}$, Shung-Tai Ho ${ }^{2,3}$, Chih-Cherng Lu ${ }^{4} \mathbb{( D}$, Ju-O Wang ${ }^{5}$, Te-Chun Yeh ${ }^{6}$ and Tso-Chou Lin ${ }^{2, * \mathbb{C}}$ \\ 1 Department of Anesthesiology, Tungs' Taichung MetroHarbor Hospital, Taichung 43503, Taiwan; \\ hsuchehao@yahoo.com.tw \\ 2 Department of Anesthesiology, Tri-Service General Hospital, National Defense Medical Center, \\ Taipei 11490, Taiwan; shungtai0617@gmail.com \\ 3 Department of Anesthesiology, Kaohsiung Medical University Hospital, Kaohsiung Medical University, \\ Kaohsiung 80756, Taiwan \\ 4 Department of Anesthesiology, Taipei Veterans General Hospital, National Defense Medical Center, \\ Taipei 11217, Taiwan; chihchernglu510803@gmail.com \\ 5 School of Public Health, National Defense Medical Center, Taipei 11490, Taiwan; carolwamg@gmail.com \\ 6 Development and Planning Center, Taipei City Hospital, Taipei 10341, Taiwan; chun0417@gmail.com \\ * Correspondence: tclin@mail.ndmctsgh.edu.tw
}

check for

updates

Citation: Hsu, C.-H.; Ho, S.-T.; Lu, C.-C.; Wang, J.-O.; Yeh, T.-C.; Lin,

T.-C. Discrepant End-Tidal

Concentrations of Sevoflurane at the

Same A-Line Autoregressive Index Level during Induction of General Anesthesia: An Observational Study. J. Clin. Med. 2021, 10, 4526. https:// doi.org/10.3390/jcm10194526

Academic Editor: Young-Tae Jeon

Received: 8 August 2021

Accepted: 27 September 2021

Published: 30 September 2021

Publisher's Note: MDPI stays neutral with regard to jurisdictional claims in published maps and institutional affiliations.

Copyright: (c) 2021 by the authors. Licensee MDPI, Basel, Switzerland. This article is an open access article distributed under the terms and conditions of the Creative Commons Attribution (CC BY) license (https:// creativecommons.org/licenses/by/ $4.0 /)$.

\begin{abstract}
Background: The A-Line Autoregressive Index (AAI), which is derived from auditory evoked potentials, has been used for determining anesthetic depth. This study verified the correlation between AAI values and the corresponding end-tidal concentrations of sevoflurane during general anesthesia induction. Methods: Thirty young male adults undergoing elective minor orthopedic surgery were sequentially allocated to receive inspiratory $3 \%, 5 \%$, or $6 \%$ sevoflurane for mask induction, followed by mechanical ventilation after tracheal intubation. The inspiratory, end-tidal and estimated jugular bulb concentrations of sevoflurane were recorded at three target AAI values: below 20, below 10, and at the start of burst suppression. Results: The mean time to loss of consciousness in the $6 \%$ sevoflurane group was shorter than that in the $5 \%$ and $3 \%$ groups; however, the groups had comparable AAI values (range: 16-45). The 6\% group had a higher end-tidal concentration $(4.5 \% \pm 0.2 \%$ vs. $3.8 \% \pm 0.2 \%, p<0.05)$ than did the $5 \%$ group, despite having the same target anesthetic levels by AAI score $\leq 10$, whereas the estimated jugular bulb concentrations were comparable (1.9\% vs. $1.9 \%$ ) in both groups. Conclusions: Following mechanical ventilation with inspiratory $3 \%, 5 \%$, or $6 \%$ sevoflurane, the end-tidal concentrations were discrepant at the same end points of AAI levels, despite similar estimated jugular bulb concentrations of sevoflurane. Thus, conventional alveolar concentration may overestimate anesthesia depth during rapid wash-in of sevoflurane.
\end{abstract}

Keywords: A-line Autoregressive Index (AAI); end-tidal concentration; general anesthesia; induction; sevoflurane

\section{Introduction}

Use of the A-Line Autoregressive Index (AAI) by autoregressive modelling with exogenous input (ARX-model) enables quicker extraction of middle-latency auditory evoked potentials [1]. In the past two decades, the AAI has been applied to differentiate degrees of anesthetic effects [2], including the correlation with the end-tidal concentrations of sevoflurane [3] and desflurane [4]. The predictable and dose-dependent response in a graded, reversible manner to minimal alveolar concentrations of volatile anesthetics provides a window to the brain for the assessment of matters such as potential awareness, burst suppression [2], and adjustment of anesthesia depth [5]. 
For inhalational anesthetics, however, a time lag between end-tidal and brain concentrations has been identified and physiologically modelled through measurement of the inspiratory, end-tidal, and jugular bulb concentrations [6-8]. Accordingly, the body's uptake and elimination [9] of anesthetics across the alveolar membrane and blood-brain barrier depend on the partition coefficient [10-12], ventilation [13], and cardiac output [7]. Sevoflurane requires nearly $40 \mathrm{~min}$ to achieve a pharmacokinetic equilibrium between arterial and jugular bulb concentrations, indicating that no further brain uptake of inspiratory $3.5 \%$ sevoflurane occurs after general anesthesia induction [7].

Clinically, using a higher inspiratory concentration of anesthetic could allow practitioners to adjust and achieve the target anesthetic level more rapidly and obtain a higher end-tidal concentration. During the wash-in period of anesthesia, data regarding the time delay between end-tidal concentrations [14] and their electroencephalographic effects, as revealed by the modern AAI, are scarce [5]. Therefore, this study examined the clinical correlations between AAI values following mechanical ventilation with different inspiratory concentrations of sevoflurane and their corresponding inspiratory and end-tidal (alveolar) concentrations during induction of general anesthesia.

\section{Materials and Methods}

\subsection{Participants}

After obtaining approval from the relevant institutional review board (TSGHIRB096-05-0082) and informed consent from each patient, the current study enrolled male patients classified as American Society of Anesthesiologists physical status I who were undergoing elective minor orthopedic surgery. Those with coexisting obesity or hypertension were excluded.

\subsection{Anesthetic Procedures}

Thirty non-premedicated young male patients were sequentially allocated to three groups: inspiratory $3 \%, 5 \%$, or $6 \%$ sevoflurane. Before mask ventilation, the anesthetic circuit was prewashed for 3 min with $3 \%, 5 \%$, or $6 \%$ sevoflurane in $6 \mathrm{~L} / \mathrm{min}$ oxygen. Under standard monitoring, the patients in each group were encouraged to take a deep breath of sevoflurane through an ordinary face mask, followed by normal spontaneous breathing or assisted mask ventilation for $5 \mathrm{~min}$. After loss of consciousness (no response to verbal command), rocuronium $(0.6 \mathrm{mg} / \mathrm{kg})$ was administered for oral tracheal intubation. Next, mechanical normoventilation (tidal volume $6-8 \mathrm{~mL} / \mathrm{kg}$ and respiratory rate $10-16 / \mathrm{min}$ to keep end-tidal $\mathrm{CO}_{2} 35-45 \mathrm{mmHg}$ ) was initiated with inspiratory $3 \%$, $5 \%$, or $6 \%$ sevoflurane in $6 \mathrm{~L} / \mathrm{min}$ oxygen for a maximum of $60 \mathrm{~min}$ or until the target sedation levels were achieved before initiating surgery. Hemodynamic variables, including heart rate, blood pressure, pulse oximetry, and capnography, were recorded at the start of induction and at $5,10,15,20,30,40,50$, and $60 \mathrm{~min}$, and inspiratory and end-tidal concentrations of sevoflurane were measured using side-stream gas sampling with a CardioCap 5 monitor (Datex, Helsinki, Finland). Intravenous bolus of nitroglycerin (50-100 mcg) for maintaining a systolic blood pressure below $160 \mathrm{mmHg}$ during tracheal intubation and ephedrine (5-10 $\mathrm{mg}$ ) for maintaining a subsequent mean blood pressure above $60 \mathrm{mmHg}$ were incrementally administered, as required. No other intravenous medications were administered during the study period.

\subsection{Data Acquisition}

An auditory evoked potential (AEP) monitor (AEP Monitor/2; version 1.6; Danmeter A/S, Odense, Denmark) was applied to the patients' forehead with contact impedance $<5 \mathrm{~K} \mathrm{Ohm}$. Headphones connected to the device provided auditory stimulus, an intermittent click $(6 \mathrm{~Hz}$ in frequency with automatically controlled volume according to the appropriate AEP extraction quality conditions). Processing time for the AAI was $30 \mathrm{~s}$ for the first detected signal, with a total update delay of $6 \mathrm{~s}$. The awake AAI level before anesthesia was reset to a maximal 60 value for all patients. An AAI range of 0-60 is rec- 
ommended to minimize oscillations while the patient is awake (high AAI values) and to provide higher-quality graphical resolutions when the patient is asleep or anesthetised (low AAI values) [15]. The monitor included a burst suppression indicator to indicate periods when the electroencephalogram (EEG) was isoelectric. The AEP monitor indicated the percentage of burst suppression over the preceding $30 \mathrm{~s}$ of the EEG signal. After loss of consciousness, the aforementioned hemodynamic and inhalation parameters were also collected at three target AAI levels: below 20, below 10, and at the beginning of burst suppression. The estimated jugular bulb concentrations of sevoflurane were calculated using the end-tidal concentrations in this study and the calculated ratios of jugular bulb over end-tidal concentrations from a related pharmacokinetic study of sevoflurane brain uptake [7]. The ratios were 0.378 at $5 \mathrm{~min}, 0.461$ at $10 \mathrm{~min}, 0.503$ at $20 \mathrm{~min}, 0.550$ at $30 \mathrm{~min}$, and 0.635 at $60 \mathrm{~min}$ following mechanical ventilation.

\subsection{Data Analysis}

Statistical analysis was conducted using SPSS version 22 (IBM Corp., Armonk, NY, USA). Descriptive statistical analyses were performed to calculate the means and standard deviations of demographic and clinical variables. Since the data did not satisfy the assumptions of equality of variance and normal distribution, the Kruskal-Wallis test and Mann-Whitney U-test were employed to analyse the change in values for intergroup comparison. When a significant difference was observed between the groups, Dunn's test was performed to identify the deviating group. Differences with $p<0.05$ were considered statistically significant.

\section{Results}

\subsection{Participant Recruitment}

Thirty adult male patients undergoing elective minor orthopedic surgery were sequentially allocated to three groups of inspiratory $3 \%, 5 \%$, or $6 \%$ sevoflurane for induction of general anesthesia.

\subsection{General Characteristics of Participants}

The general data are presented in Table 1 . The $6 \%$ sevoflurane group had a shorter time to loss of consciousness but similar AAI values relative to the $5 \%$ and $3 \%$ groups. The awake AAI level before anesthesia was reset to 60 for each patient.

Table 1. Demographic data.

\begin{tabular}{ccccc}
\hline & Sevoflurane 3\% & Sevoflurane 5\% & Sevoflurane 6\% & $p$ Value \\
\hline Patient number & 10 males & 10 males & 10 males & \\
Age, year & $21.9 \pm 1.2$ & $21.7 \pm 1.8$ & $21.9 \pm 1.4$ & 0.956 \\
Height, cm & $175.3 \pm 8.4$ & $169.9 \pm 2.7$ & $171.3 \pm 4.7$ & 0.293 \\
Weight, kg & $71.2 \pm 10.8$ & $64.8 \pm 12.1$ & $62.8 \pm 9.3$ & 0.206 \\
Body mass index, & $23.2 \pm 3.7$ & $22.4 \pm 4.1$ & $21.3 \pm 2.0$ & 0.42 \\
kg/m & $122.5 \pm 35.0$ & $89.5 \pm 22.5$ & $71.5 \pm 12.5$ & 0.002 \\
Time to & $(75-180)$ & $(55-120)$ & $(55-90)$ & \\
loss of consciousness, & $31.7 \pm 9.9$ & $26.2 \pm 7.0$ & $28.6 \pm 6.8$ & 0.355 \\
sec & $(16-45)$ & $(20-36)$ & $(20-41)$ & \\
AAI values at & &
\end{tabular}

Data are presented as the mean \pm standard deviation (range). AAI, A-Line Autoregressive Index.

\subsection{Iinspiratory and End-Tidal Concentrations of Sevoflurane}

Figure 1 illustrates the pharmacokinetics of inspiratory and end-tidal concentrations of sevoflurane in the three groups. The administration of sevoflurane in the $6 \%$ group was terminated at $30 \mathrm{~min}$ after burst suppression status had been achieved. 


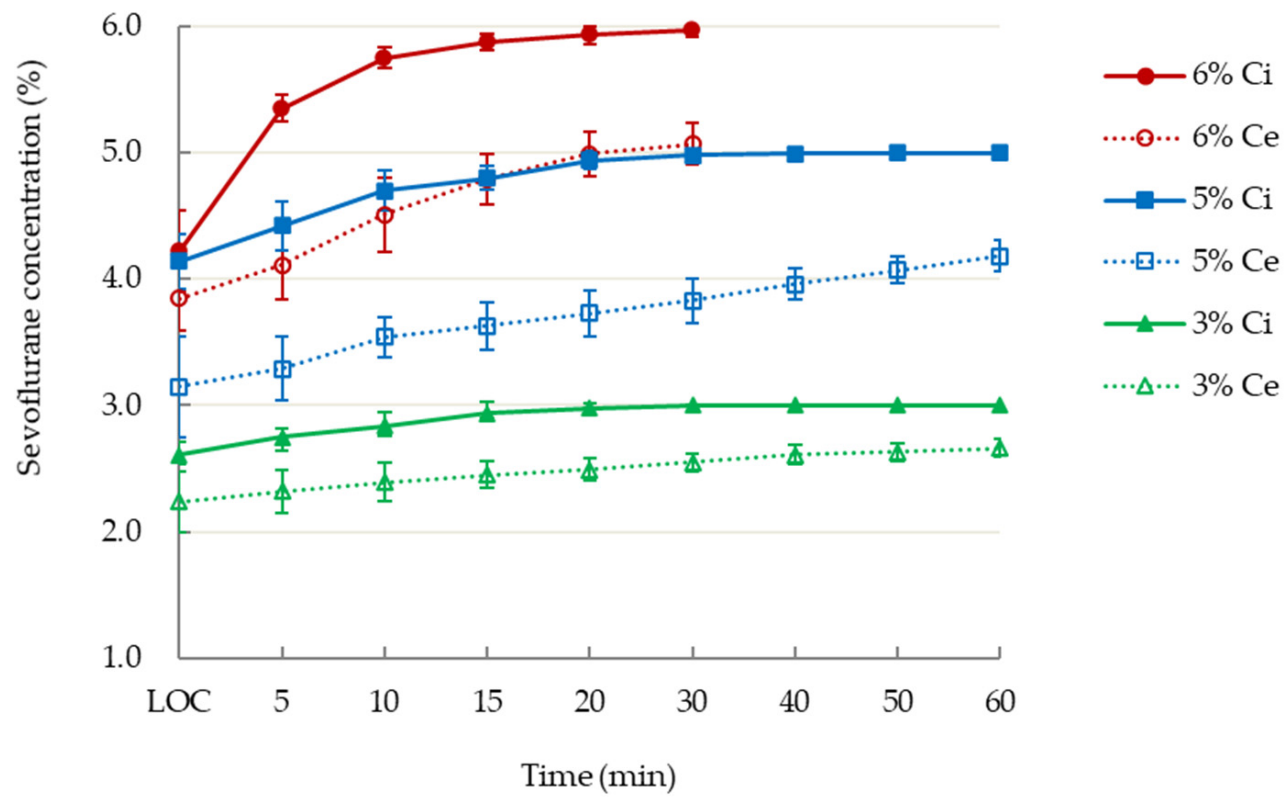

Figure 1. T Inspiratory (Ci) and end-tidal (Ce) concentrations of inspiratory 3\%, 5\%, or 6\% sevoflurane following loss of consciousness (LOC) during administration of general anesthesia.

\subsection{AAI Values and Sevoflurane Concentrations}

The time required to reach the programmed AAI levels and their corresponding inspiratory, end-tidal and estimated jugular bulb concentrations are summarised in Table 2. The AAI scores in the 3\% sevoflurane group did not fall below 10 during the $60-\mathrm{min}$ period (14.6 \pm 2.3 at $60 \mathrm{~min}$ ). The $6 \%$ sevoflurane group were terminated at $30 \mathrm{~min}$ on account of low AAI scores $(0.9 \pm 2.8)$ and burst suppression. At the same AAI end points, including values $\leq 20$, values $\leq 10$, and at the start of burst suppression, the mean end-tidal concentrations in the $6 \%$ sevoflurane group were significantly higher than those in the $5 \%$ sevoflurane group, whereas the estimated jugular bulb concentrations were comparable between these two groups. The estimated jugular bulb concentration at $30 \mathrm{~min}$ in the $6 \%$ group was twice that in the $3 \%$ group $(2.8 \% \pm 0.1 \%$ vs. $1.4 \% \pm 0.1 \%$, respectively).

Table 2. Time required to achieve the target A-Line Autoregressive Index (AAI) values and the corresponding inspiratory, end-tidal and estimated jugular bulb concentrations of sevoflurane.

\begin{tabular}{|c|c|c|c|c|c|}
\hline Anesthesia Level & $\begin{array}{l}\text { Sevoflurane } \\
\text { Group }\end{array}$ & $\begin{array}{l}\text { Time } \\
\text { after Induction }\end{array}$ & $\begin{array}{c}\text { Inspiratory } \\
\text { Concentration } \\
(\%)\end{array}$ & $\begin{array}{c}\text { End-Tidal } \\
\text { Concentration } \\
(\%)\end{array}$ & $\begin{array}{c}\text { Estimated Jugular } \\
\text { Bulb Concentration } \\
(\%)\end{array}$ \\
\hline \multirow{3}{*}{$\begin{array}{c}\text { Loss of } \\
\text { consciousness }\end{array}$} & $3 \%$ & $122.5 \pm 35.0 \mathrm{~s}$ & $2.6 \pm 0.1$ & $2.2 \pm 0.2$ & \\
\hline & $5 \%$ & $89.5 \pm 22.5 \mathrm{~s}$ & $4.1 \pm 0.2$ & $3.2 \pm 0.4$ & \\
\hline & $6 \%$ & $71.5 \pm 12.5 \mathrm{~s}$ & $4.2 \pm 0.3$ & $3.9 \pm 0.3$ & \\
\hline \multirow{3}{*}{$\mathrm{AAI} \leq 20$} & $3 \%$ & $5.9 \pm 1.5 \mathrm{~min}$ & $2.8 \pm 0.1$ & $2.4 \pm 0.1$ & $0.9 \pm 0.0$ \\
\hline & $5 \%$ & $4.8 \pm 3.0 \mathrm{~min}$ & $4.3 \pm 0.2$ & $3.4 \pm 0.2$ & $1.3 \pm 0.1$ \\
\hline & $6 \%$ & $2.6 \pm 2.0 \mathrm{~min}$ & $4.8 \pm 0.6$ & $4.1 \pm 0.2$ & $1.4 \pm 0.1$ \\
\hline \multirow{2}{*}{$\mathrm{AAI} \leq 10$} & $5 \%$ & $21.4 \pm 9.8 \mathrm{~min}$ & $4.9 \pm 0.1$ & $3.8 \pm 0.2$ & $1.9 \pm 0.1$ \\
\hline & $6 \%$ & $8.3 \pm 2.9 \mathrm{~min}$ & $5.6 \pm 0.2$ & $4.5 \pm 0.2 *$ & $1.9 \pm 0.1$ \\
\hline Start of & $5 \%$ & $26.8 \pm 11.1 \mathrm{~min}$ & $4.9 \pm 0.1$ & $3.9 \pm 0.1$ & $2.1 \pm 0.1$ \\
\hline burst suppression & $6 \%$ & $9.9 \pm 2.6 \mathrm{~min}$ & $5.7 \pm 0.1$ & $4.7 \pm 0.1^{\#}$ & $2.2 \pm 0.1$ \\
\hline
\end{tabular}


Table 2. Cont.

\begin{tabular}{cccccc}
\hline Anesthesia Level & $\begin{array}{c}\text { Sevoflurane } \\
\text { Group }\end{array}$ & $\begin{array}{c}\text { Time } \\
\text { after Induction }\end{array}$ & $\begin{array}{c}\text { Inspiratory } \\
\text { Concentration } \\
\mathbf{( \% )}\end{array}$ & $\begin{array}{c}\text { End-Tidal } \\
\text { Concentration } \\
\mathbf{( \% )}\end{array}$ & $\begin{array}{c}\text { Estimated Jugular } \\
\text { Bulb Concentration } \\
\mathbf{( \% )}\end{array}$ \\
\hline \multirow{2}{*}{ At 30 $\mathrm{min}$} & $3 \%$ & & $3.0 \pm 0.0$ & $2.6 \pm 0.1$ & $1.4 \pm 0.0$ \\
& $5 \%$ & $30 \mathrm{~min}$ & $5.0 \pm 0.0$ & $3.8 \pm 0.2$ & $2.1 \pm 0.1$ \\
\hline \multirow{2}{*}{ At $60 \mathrm{~min}$} & $6 \%$ & & $6.0 \pm 0.0$ & $5.1 \pm 0.2$ & $2.8 \pm 0.1$ \\
\hline
\end{tabular}

Data are presented as the mean \pm standard deviation. AAI, A-Line Autoregressive Index. The estimated jugular bulb concentrations of sevoflurane were calculated using the ratios of jugular bulb over end-tidal concentrations in the previous pharmacokinetic study of sevoflurane uptake during induction [7]. * and ${ }^{\#}, p$ value $<0.001$, as compared with the end-tidal concentrations in the $5 \%$ group.

\subsection{Hemodynamic Variables during Induction}

Table 3 provides the comparable hemodynamic variables. Three patients in the $5 \%$ group and three patients in the $6 \%$ group received intermittent ephedrine $5 \mathrm{mg}$ ( $\max 15 \mathrm{mg}$ in total) to maintain their mean arterial blood pressure above $60 \mathrm{mmHg}$. None of the patients experienced adverse events.

Table 3. Hemodynamic variables.

\begin{tabular}{|c|c|c|c|c|}
\hline Group & Time & $\begin{array}{c}\text { Heart Rate } \\
\text { (beats per min) }\end{array}$ & $\begin{array}{l}\text { Mean Blood Pressure } \\
(\mathrm{mmHg})\end{array}$ & $\begin{array}{c}\text { End-Tidal } \mathrm{CO}_{2} \\
(\mathrm{mmHg})\end{array}$ \\
\hline \multirow{10}{*}{$\begin{array}{c}3 \% \\
\text { sevoflurane }\end{array}$} & Before induction & $65.2 \pm 8.7$ & $86.9 \pm 7.0$ & - \\
\hline & Loss of consciousness & $64.7 \pm 7.1$ & $82.6 \pm 8.2$ & $40.4 \pm 2.7$ \\
\hline & $5 \mathrm{~min}$ & $87.3 \pm 22.2$ & $89.1 \pm 16.5$ & $41.7 \pm 3.1$ \\
\hline & $10 \mathrm{~min}$ & $71.4 \pm 16.7$ & $73.4 \pm 12.6$ & $42.3 \pm 2.0$ \\
\hline & $15 \min$ & $69.1 \pm 13.8$ & $71.6 \pm 8.6$ & $42.7 \pm 1.8$ \\
\hline & $20 \mathrm{~min}$ & $66.1 \pm 12.5$ & $69.5 \pm 8.8$ & $43.0 \pm 1.1$ \\
\hline & $30 \mathrm{~min}$ & $66.6 \pm 11.8$ & $68.9 \pm 10.5$ & $43.2 \pm 1.4$ \\
\hline & $40 \mathrm{~min}$ & $66.6 \pm 12.9$ & $67.7 \pm 10.4$ & $43.4 \pm 1.3$ \\
\hline & $50 \mathrm{~min}$ & $65.1 \pm 12.4$ & $66.4 \pm 8.5$ & $43.9 \pm 1.4$ \\
\hline & $60 \mathrm{~min}$ & $63.2 \pm 12.4$ & $65.9 \pm 8.6$ & $44.1 \pm 2.0$ \\
\hline \multirow{10}{*}{$\begin{array}{c}5 \% \\
\text { sevoflurane }\end{array}$} & Before induction & $72.9 \pm 11.7$ & $86.3 \pm 9.9$ & - \\
\hline & Loss of consciousness & $69.1 \pm 11.7$ & $81.0 \pm 10.4$ & $35.0 \pm 2.6$ \\
\hline & $5 \mathrm{~min}$ & $92.0 \pm 20.4$ & $79.7 \pm 8.5$ & $35.2 \pm 2.7$ \\
\hline & $10 \mathrm{~min}$ & $82.7 \pm 14.7$ & $74.4 \pm 8.8$ & $35.6 \pm 3.1$ \\
\hline & $15 \mathrm{~min}$ & $82.0 \pm 17.6$ & $70.6 \pm 6.9$ & $36.1 \pm 3.4$ \\
\hline & $20 \mathrm{~min}$ & $79.0 \pm 16.3$ & $69.4 \pm 6.0$ & $37.6 \pm 4.0$ \\
\hline & $30 \mathrm{~min}$ & $83.4 \pm 20.0$ & $69.6 \pm 10.0$ & $38.7 \pm 4.5$ \\
\hline & $40 \mathrm{~min}$ & $83.7 \pm 21.6$ & $65.2 \pm 3.3$ & $39.4 \pm 5.1$ \\
\hline & $50 \mathrm{~min}$ & $78.0 \pm 20.4$ & $63.0 \pm 2.8$ & $40.0 \pm 5.2$ \\
\hline & $60 \mathrm{~min}$ & $73.4 \pm 17.5$ & $61.1 \pm 2.8$ & $41.5 \pm 5.7$ \\
\hline \multirow{7}{*}{$\begin{array}{c}6 \% \\
\text { sevoflurane }\end{array}$} & Before induction & $72.7 \pm 14.7$ & $86.8 \pm 10.6$ & - \\
\hline & Loss of consciousness & $75.1 \pm 22.4$ & $80.6 \pm 9.4$ & $38.3 \pm 3.4$ \\
\hline & $5 \min$ & $93.6 \pm 22.7$ & $75.3 \pm 13.9$ & $36.7 \pm 2.9$ \\
\hline & $10 \mathrm{~min}$ & $80.6 \pm 14.0$ & $69.4 \pm 13.1$ & $35.9 \pm 2.0$ \\
\hline & $15 \min$ & $81.4 \pm 9.4$ & $65.4 \pm 5.0$ & $36.1 \pm 1.4$ \\
\hline & $20 \mathrm{~min}$ & $80.3 \pm 12.0$ & $62.8 \pm 4.1$ & $36.8 \pm 2.3$ \\
\hline & $30 \mathrm{~min}$ & $79.4 \pm 13.8$ & $63.3 \pm 6.5$ & $38.0 \pm 2.2$ \\
\hline
\end{tabular}

Data are presented as the mean \pm standard deviation. 


\section{Discussion \\ 4.1. Main Findings}

By using AAI monitoring, we demonstrated that, relative to patients receiving inspiratory $5 \%$ or $3 \%$ sevoflurane, those receiving inspiratory $6 \%$ sevoflurane had a shorter time to loss of consciousness with comparable AAI values and a shorter time required to reach the target AAI values under mechanical ventilation but higher end-tidal concentrations at the same end-point AAI values. The estimated jugular bulb concentrations were similar between the $6 \%$ and $5 \%$ groups at the same AAI levels. These findings indicate that alveolar concentration may overestimate anesthesia depth because of a notable gap between end-tidal and brain concentrations during rapid wash-in of a higher concentration of sevoflurane.

\subsection{Higher Inspiratory and End-Tidal Concentrations at the Same AAI Values during Wash-In}

The minimum alveolar concentration (MAC) required to prevent movement in response to surgical incision in 50\% of patients has been used as an indicator of anesthesia depth of inhalational anesthetics for decades [16]. This value is derived from a steady end-tidal concentration representing the arterial and brain concentrations of the anesthesia. Lu et al. [7] demonstrated that the ratio of arterial/end-tidal concentration of sevoflurane remained at 0.63 after $30 \mathrm{~min}$ of mechanical ventilation with inspiratory $3.5 \%$ sevoflurane. They also revealed that a higher inspiratory concentration of isoflurane could accelerate its brain uptake [8]. Equilibrium between arterial and jugular bulb concentrations was achieved after $40 \mathrm{~min}$ for inspiratory $2 \%$ isoflurane and $50 \mathrm{~min}$ for $1 \%$ isoflurane [8]. The arterial and jugular bulb concentrations in the $2 \%$ isoflurane group were approximately double those of the $1 \%$ isoflurane group [8]. In the current study, a similar pharmacokinetic phenomenon was observed in estimated jugular bulb concentrations at $30 \mathrm{~min}(2.8 \% \mathrm{vs}$. $1.4 \%$ ) between the $6 \%$ and $3 \%$ sevoflurane groups. As expected, the time to loss of consciousness and the time required to achieving the target AAI values were shorter in the $6 \%$ sevoflurane group. Theoretically, end-tidal concentrations for calculating MAC values as anesthesia depth should be similar at the same target AAI levels regardless of whether inspiratory $5 \%$ or $6 \%$ sevoflurane is used. However, the actual end-tidal concentrations were higher in the $6 \%$ group at the same AAI values: $\leq 20, \leq 10$, and at the start of burst suppression. The estimated jugular bulb concentrations were similar at the same end points of AAI values between the $6 \%$ and $5 \%$ sevoflurane groups, which is consistent with our previous observations regarding the pharmacokinetics of sevoflurane uptake [7]. This finding indicates a lag in the time required to achieve transition of anesthesia across the alveolar membrane and blood-brain barrier, [17] which is determined by various blood/gas and brain/blood partition coefficients of inhalational anesthetics [18]. The MAC value might be overestimated as a result of higher end-tidal concentration during wash-in with a higher inspiratory concentration of anesthetics, which may result in de facto inadequate anesthesia depth under surgical stimulation.

\subsection{EEG Processing for Adjustment of Anesthesia Depth}

A composite AEP index incorporating EEG has been used as an indicator for anesthesia depth according to dose [2] and in relation to age, [3] which is a more discriminant predictor of different clinical states of general anesthesia [19]. However, some studies have failed to observe a graded response with steady-state end-tidal concentrations of sevoflurane, neither decreasing from $2 \%$ to $1.5 \%$ and $1 \%$ in adults [20] nor increasing from $1.5 \%$ to $2 \%$ and $2.5 \%$ in infants and children [21]. The so-called steady-state is based on the constant administration of an end-tidal concentration of sevoflurane for $11 \mathrm{~min}$ [20] and was calculated using the Gas Man Anesthesia Simulator programme for equilibration of partial pressures between the brain and the lungs [22]. Our previous pharmacokinetic study, which employed blood sampling for sevoflurane concentration analysis, demonstrated that the time required to achieve equilibration between arterial and jugular bulb concentrations (no further brain uptake) was $38.5 \mathrm{~min}$ following mechanical ventilation with inspiratory 
$3.5 \%$ sevoflurane, and a near constant end-tidal concentration was achieved after $30 \mathrm{~min}$ of ventilation [7]. Therefore, the early change in end-tidal concentrations during wash-in or wash-out may not accurately reflect the true brain uptake and anesthesia depth. The application of EEG processing could facilitate the adjustment of anesthesia depth during the wash-in or wash-out periods.

\subsection{AAI Values and MAC of Sevoflurane}

The AAI values in the 3\% sevoflurane group did not decrease to less than 10 during 60 min of ventilation despite the ultimate end-tidal concentration being $2.7 \%$. Young male patients were recruited for this study to reduce the impact of interindividual comorbidities and age or sex-related variability on MAC values [23]. One MAC of sevoflurane is $1.8 \%$ at the age of 40 years, with an approximate decrease of $6 \%$ every decade [23]. Women appear to have the same MAC as men [24]. However, AAI values were reported to be severely attenuated or reach a value of 0 under a $2 \%$ steady-state end-tidal concentration of sevoflurane in women aged 20-60 years [25].

\subsection{Limitations}

Two limitations of the current study should be addressed. First, all 30 patients were young male patients aged 20-25 years. The small sample size and the inclusion of only young men in this observational study may have limited the clinical application of the findings; future research could include a more diverse sample. Second, AAI values only indicated the sedation levels before surgery in our patients and not the clinical anesthesia depth during surgery. Possible confounding factors that could interfere with EEG processing were excluded, such as surgical stimulation, noisy environment, hypoglycemia, cerebral ischemia and neurological disorders, [1] and intravenous benzodiazepine and propofol.

\section{Conclusions}

We demonstrated that patients receiving a higher sevoflurane concentration had a shorter time to loss of consciousness with comparable AAI values and had higher end-tidal concentrations of sevoflurane at the same end-point AAI levels. Their estimated jugular bulb concentrations were also similar at the same AAI levels. Anesthesia depth, calculated according to alveolar concentration, may be overestimated during the rapid wash-in of inhalation anesthesia. This discrepancy between alveolar and brain concentrations at the same AAI values of anesthesia should be considered by practitioners.

Author Contributions: Conceptualization, S.-T.H. and C.-C.L.; methodology, S.-T.H. and C.-H.H.; Data curation and formal analysis, J.-O.W., T.-C.Y. and T.-C.L.; investigation, C.-H.H. and C.-C.L.; visualization, T.-C.L.; writing-original draft, C.-H.H. and T.-C.L.; writing-reviewing and editing, S.-T.H., J.-O.W. and T.-C.L.; project administration, S.-T.H.; funding acquisition, S.-T.H. and T.-C.L. All authors have read and agreed to the published version of the manuscript.

Funding: This research was funded by Tri-Service General Hospital, grant number TSGH-D-109192.

Institutional Review Board Statement: The study was conducted according to the guidelines of the Declaration of Helsinki and approved by the Institutional Review Board of Tri-Service General Hospital (protocol code TSGHIRB-096-05-0082).

Informed Consent Statement: Informed consent was obtained from all subjects involved in the study.

Data Availability Statement: The data presented in this study are available on request from the corresponding author.

Conflicts of Interest: The authors declare no conflict of interest. 


\section{References}

1. Bell, S.L.; Smith, D.C.; Allen, R.; Lutman, M.E. Recording the middle latency response of the auditory evoked potential as a measure of depth of anaesthesia. A technical note. Br. J. Anaesth. 2004, 92, 442-445. [CrossRef] [PubMed]

2. Horn, B.; Pilge, S.; Kochs, E.F.; Stockmanns, G.; Hock, A.; Schneider, G. A combination of electroencephalogram and auditory evoked potentials separates different levels of anesthesia in volunteers. Anesth. Analg. 2009, 108, 1512-1521. [CrossRef] [PubMed]

3. Feuerecker, M.; Lenk, M.; Flake, G.; Edelmann-Gahr, V.; Wiepcke, D.; Hornuss, C.; Daunderer, M.; Müller, H.H.; Kuhnle, G.E. Effects of increasing sevoflurane MAC levels on mid-latency auditory evoked potentials in infants, schoolchildren, and the elderly. Br. J. Anaesth. 2011, 107, 726-734. [CrossRef]

4. Kreuer, S.; Bruhn, J.; Larsen, R.; Buchinger, H.; Wilhelm, W. A-line, bispectral index, and estimated effect-site concentrations: A prediction of clinical end-points of anesthesia. Anesth. Analg. 2006, 102, 1141-1146. [CrossRef] [PubMed]

5. Anderson, R.E.; Barr, G.; Assareh, H.; Jakobsson, J. The AAI index, the BIS index and end-tidal concentration during wash in and wash out of sevoflurane. Anaesthesia 2003, 58, 531-535. [CrossRef]

6. Lu, C.C.; Tsai, C.S.; Ho, S.T.; Chueng, C.M.; Wang, J.J.; Wong, C.S.; Chang, S.Y.; Lin, C.Y. Pharmacokinetics of desflurane uptake into the brain and body. Anaesthesia 2004, 59, 216-221. [CrossRef] [PubMed]

7. Lu, C.C.; Tsai, C.S.; Ho, S.T.; Chen, W.Y.; Wong, C.S.; Wang, J.J.; Hu, O.Y.; Lin, C.Y. Pharmacokinetics of sevoflurane uptake into the brain and body. Anaesthesia 2003, 58, 951-956. [CrossRef]

8. Lu, C.C.; Ho, S.T.; Wang, J.J.; Wong, C.S.; Hu, O.Y.; Chang, S.Y.; Lin, C.Y. Pharmacokinetics of isoflurane: Uptake in the brain. Pharmacology 2003, 69, 102-107. [CrossRef]

9. Lu, C.C.; Lin, T.C.; Hsu, C.H.; Tsai, C.S.; Sheen, M.J.; Hu, O.Y.; Ho, S.T. Pharmacokinetics of sevoflurane elimination from respiratory gas and blood after coronary artery bypass grafting surgery. J. Anesth. 2014, 28, 873-879. [CrossRef]

10. Lin, T.C.; Lu, C.C.; Hsu, C.H.; Wu, G.J.; Lee, M.S.; Ho, S.T. Duration effect of desflurane anesthesia and its awakening time and arterial concentration in gynecologic patients. Clinics 2013, 68, 1305-1311. [CrossRef]

11. Lin, T.C.; Lu, C.C.; Hsu, C.H.; Su, H.Y.; Lee, M.S.; Ho, S.T. Arterial blood and end-tidal concentrations of sevoflurane during the emergence from anesthesia in gynecologic patients. Clinics 2015, 70, 196-201. [CrossRef]

12. Lin, T.C.; Lu, C.C.; Hsu, C.H.; Pergolizz, J.V., Jr.; Chang, C.C.; Lee, M.S.; Ho, S.T. Awakening arterial blood and end-tidal concentrations of isoflurane in female surgical patients. Medicine 2016, 95, e4370. [CrossRef] [PubMed]

13. Lu, C.C.; Lin, T.C.; Hsu, C.H.; Yu, M.H.; Ku, C.H.; Chen, T.L.; Chen, R.M.; Ho, S.T. Hyperventilation accelerates rise in arterial blood concentrations of sevoflurane in gynecologic patients. J. Anesth. 2013, 27, 35-42. [CrossRef] [PubMed]

14. Lin, T.C.; Lu, C.C.; Li, C.Y.; Chang, C.C.; Ho, S.T. Arterial blood concentration of sevoflurane during single-breath induction and tracheal intubation in gynecologic patients. J. Clin. Anesth. 2008, 20, 496-500. [CrossRef] [PubMed]

15. Sinha, P.; Koshy, T. Monitoring devices for measuring the depth of anaesthesia-An overview. Indian J. Anaesth. $2007,51,365$.

16. Eger, E.I., 2nd; Saidman, L.J.; Brandstater, B. Minimum alveolar anesthetic concentration: A standard of anesthetic potency. Anesthesiology 1965, 26, 756-763. [CrossRef]

17. Rehberg, B.; Bouillon, T.; Zinserling, J.; Hoeft, A. Comparative pharmacodynamic modeling of the electroencephalographyslowing effect of isoflurane, sevoflurane, and desflurane. Anesthesiology 1999, 91, 397-405. [CrossRef]

18. Yasuda, N.; Targ, A.G.; Eger, E.I., 2nd. Solubility of I-653, sevoflurane, isoflurane, and halothane in human tissues. Anesth. Analg. 1989, 69, 370-373. [CrossRef]

19. Jeleazcov, C.; Schneider, G.; Daunderer, M.; Scheller, B.; Schuttler, J.; Schwilden, H. The discriminant power of simultaneous monitoring of spontaneous electroencephalogram and evoked potentials as a predictor of different clinical states of general anesthesia. Anesth. Analg. 2006, 103, 894-901. [CrossRef]

20. Alpiger, S.; Helbo-Hansen, H.S.; Jensen, E.W. Effect of sevoflurane on the mid-latency auditory evoked potentials measured by a new fast extracting monitor. Acta Anaesthesiol. Scand. 2002, 46, 252-256. [CrossRef]

21. Ironfield, C.M.; Davidson, A.J. AEP-monitor/2 derived, composite auditory evoked potential index (AAI-1.6) and bispectral index as predictors of sevoflurane concentration in children. Paediatr. Anaesth. 2007, 17, 452-459. [CrossRef] [PubMed]

22. Philip, J.H. Gas Man-An example of goal oriented computer-assisted teaching which results in learning. Int. J. Clin. Monit. Comput. 1986, 3, 165-173. [CrossRef]

23. Mapleson, W.W. Effect of age on MAC in humans: A meta-analysis. Br. J. Anaesth. 1996, 76, 179-185. [CrossRef]

24. Eger, E.I., 2nd; Laster, M.J.; Gregory, G.A.; Katoh, T.; Sonner, J.M. Women appear to have the same minimum alveolar concentration as men: A retrospective study. Anesthesiology 2003, 99, 1059-1061. [CrossRef] [PubMed]

25. Schwender, D.; Conzen, P.; Klasing, S.; Finsterer, U.; Poppel, E.; Peter, K. The effects of anesthesia with increasing end-expiratory concentrations of sevoflurane on midlatency auditory evoked potentials. Anesth. Analg. 1995, 81, 817-822. [CrossRef] [PubMed] 